\title{
LA GOBERNABILIDAD SOCIALISTA DE LOS ESPACIOS LITORALES: EL CASO DE CIUDAD DE LA HABANA, CUBA*
}

\author{
José Manuel Mateo Rodriguez** \\ Antonio Jeovah de Andrade Meireles*** \\ Edson Vicente da Silva**** \\ Arturo Rua de Cabo*****
}

\begin{abstract}
RESUMEN
La irrupción, a partir de comienzos de la década de los años 1960 de un proyecto socialista en Cuba, constituyó un cambio radical en la forma de gobernar las sociedades en el hemisferio occidental. Por primera vez en América Latina, un país se proponía cambiar de manera estructural los fundamentos de organización de la sociedad capitalista, y construir un proyecto de sociedad basada en el socialismo. El presente artículo, pretende exponer como se han llevado a cabo los cambios en la organización de los espacios litorales de Ciudad de La Habana, en la construcción del estilo socialista de desarrollo. Pretende en particular reflexionar sobre los desafíos, que en los inicios del siglo XXI enfrenta la sociedad cubana, para pasar a la conquista de la sostenibilidad, como fundamento para hacer más eficiente y duradera la gobernabilidad.

Al final se ha realizado una síntesis de los problemas ambientales y las posibles soluciones, vinculada a un abordaje integrado con la dinámica litoral y las distintas formas de utilización. Las sugerencias acciones a medio y largo plazo, incluso con ejemplos de acciones ya desarrolladas en otros tramos del litoral cubano.
\end{abstract}

PALABRAS-CLAVE: Evolución litoral, gobernabilidad y organización espacial del territorio.

\section{Introducción}

La irrupción, a partir de comienzos de la década de los años 1960 de un proyecto socialista en Cuba, constituyó un cambio radical en la forma de gobernar las sociedades en el hemisferio occidental. Por primera vez en América Latina, un país se proponía cambiar de manera estructural los fundamentos de organización de la sociedad capitalista, y construir un proyecto de sociedad basada en el socialismo.

Sin embargo, es conocido, que cualquier sociedad se fundamenta en su espacio, en la organización espacial, como cimiento para toda estructura social, y para todo proceso de cambio (Santos, 1996; Correa, 2002, 2007). Por lo tanto, se trataba entonces de realizar profundas transformaciones en la organización espacial, en los procesos de ocupación, asimilación y apropiación del espacio que repercutían de manera directa en la gestión territorial y ambiental. Se partía entonces, de efectuar el cambio en la organización espacial, como base de la construcción de un nuevo proyecto de gobernabilidad.

Es de destacar, que en el presente articulo, de acuerdo a Ochoa y Olsen (2007) se entiende como gobernabilidad la capacidad de una sociedad de dar la orientación y el destino de los cambios que espera, de acuerdo a su proyecto de conducción y organización. Corresponde a los fundamentos, a la base ética, a las grandes políticas que definen el tipo de sociedad que se anhela y que están en la base de los cambios que

\footnotetext{
*Este trabajo se ha realizado en el marco del convenio de colaboración entre la entidad CAPES de la República Federal do Brasil, y el Ministerio de Educación Superior de la República de Cuba.

**Prof. Dr. del Departamento de Geografía de la Universidad de la Habana (UH) - mateo@geo.uh.cu; ***Prof. Dr. del Departamento de Geografía de la Universidad Federal de Ceará (UFC) - meireles@ufc.br; ****Prof. Dr. del Departamento de Geografía de la Universidad Federal de Ceará (UFC) -
} cacau@ufc.br; *****Prof. Dr. del Departamento de Geografía de la Universidad de la Habana (UH) - arturo@geo.uh.cu 
se quieren lograr. Una manifestación directa de este concepto es la definición de gobernanza. Por ello se entiende a la capacidad operativa de construir y manejar los mecanismos y practicas que aportan y sostienen los recursos hacia los resultados esperados. Corresponde a los mecanismos y procesos concretos por medio de los cuales se organizan y guían los cambios.

Es conocido, que Cuba, como un conjunto de islas y archipiélagos, ha tenido a los espacios litorales, como un fundamento de partida en toda la organización espacial del territorio. Por lo tanto, la nueva gobernabilidad socialista ha tenido que partir en gran parte de la reorganización espacial de sus litorales, y en particular de sus litorales intensamente urbanizados, como centro neurálgico de la organización espacial de todo el país.

El presente artículo, pretende exponer como se han llevado a cabo los cambios en la organización de los espacios litorales de Ciudad de La Habana, en la construcción del estilo socialista de desarrollo. Pretende en particular reflexionar sobre los desafíos, que en los inicios del siglo XXI enfrenta la sociedad cubana, para pasar a la conquista de la sostenibilidad, como fundamento para hacer más eficiente y duradera la gobernabilidad socialista.

\section{La Habana y los espacios litorales cubanos}

Cuba, constituida por dos islas de tamaño relativamente considerable (Cuba e Isla de Pinos, actualmente conocida como Isla de la Juventud), está formada además por cerca de 4000 pequeñas islas, conocidas como "cayos". Su insularidad, la amplia presencia de la línea costera, por mas de 5000 kilómetros, la relativa reducción de la diferencia en distancia entre las costas norte y sur; la permanente presencia del mar; la existencia de bahías cerradas de significativa profundidad; y la presencia de recursos pesqueros en sus plataformas circundantes; además de la situación privilegiada entre el Caribe y el Golfo de México, fueron todos ellos factores significativos en la localización, ubicación y organización espacial de los objetos geográficos en todo el proceso de su colonización y asimilación económica y humana.

La ciudad de La Habana, actualmente con algo más de dos millones de habitantes, para un país de cerca de 12 millones, ha sido el centro político, económico y cultural del país. Situada en la parte nor occidental de la Isla de Cuba, su situación privilegiada, la presencia de una bahía profunda y cerrada, y su amplia llanura litoral circundante, continuamente aireada por los alisios y las brisas, en un entorno privilegiado por recursos agrícolas e hídricos, han constituido factores significativos en la localización del sitio como sede del principal foco de organización espacial del país. Este proceso puede resumirse en tres grandes etapas:

- La etapa colonial, de dominio español, en la que predominó la conversión de Cuba en una gran plantación, y en la que La Habana, con su función de "puerto escala", era el puente de conexión entre la metrópoli española y las colonias iberoamericanas.

- La etapa neocolonial, a partir de 1902 , en la que se re vigorizó el papel de plantación azucarera, ahora en una dependencia de los Estados Unidos, y en la que La Habana, constituyó el centro espacial neurálgico de la organización del espacio y de conexión con los comandos internacionales de dominio económico y político.

- La etapa revolucionaria, a partir de 1959, en la que se produce un cambio estructural radical en todas las facetas del quehacer económico y social, una re orientación de la explotación de los recursos, y en la que La Habana, actúa como el centro coordinador de toda esa gestión de cambios.

En toda la historia de la formación y conformación del territorio cubano, La Habana, de tal modo ha desempeñado el papel protagónico de la organización del espacio a nivel nacional, y el punto de conexión privilegiando a las relaciones con otros territorios. De tal manera, los espacios que conforman el territorio han estado sujetos a una fuerte demanda, por los diferentes agentes 
económicos y actores sociales. Cada uno de los espacios, ha hecho establecer su modelo de organización, que debía responder a las exigencias de las sociedades que lo construían.

La Revolución Cubana, tiene ante si, la inmensa tarea de reconstruir un nuevo modelo político y social, de erigir una nueva gobernabilidad, y por lo tanto de conformar un nuevo modelo espacial. Las exigencias de ese modelo social, se expondrán a continuación.

\section{La gobernabilidad socialista, y el nuevo modelo de organización espacial}

El proyecto de la Revolución Cubana, tenía los siguientes presupuestos básicos: crear una sociedad donde imperase la igualdad y justicia social; garantizar a la población las necesidades básicas con una calidad de vida digna para todos; promover la socialización de los recursos naturales y de la infraestructura económica existente.

Para garantizar esos objetivos básicos, el espacio territorial debería cumplir con los siguientes requisitos: el acceso de la población a todos sus espacios; un crecimiento significativo de la población; las necesidades de recreación; el mantenimiento y mejoramiento de la infraestructura; la optimización de los recursos y servicios ambientales.

La Revolución Cubana, heredó del régimen neocolonial una situación social caracterizada por los siguientes rasgos: significativa desigualdad social; perdida de la soberanía y dominio político del país por parte de la potencia imperial; dependencia tecnológica y cultural; sub explotación de los recursos, significativo desbalance y desigualdad territorial.

El modelo espacial neocolonial que heredó el nuevo proyecto social revolucionario, subordinado a las características del régimen político eran los siguientes: significativa segregación espacial y ambiental con la presencia de la privatización de objetos y sistemas espaciales; estructuras espaciales incompletas; objetos excesivamente artificializados que provocaron significativos impactos espaciales y ambientales; fuerte alteración de sistemas ambientales naturales estratégicos; funciones y relaciones espaciales inequilibradas y significativamente transformadas.

Teniendo en cuenta, el carácter diferenciado de los espacios del territorio, el modelo espacial, tanto el precedente como el que se construía, tenia un marcado carácter diferenciador. Esa diferenciación se ha manifestado, en las diversas funciones, a cumplir, en sus problemas y en la situación prevaleciente, pero también en los desafíos que de manera diferenciada tiene cada uno de esos grandes espacios.

\section{Los espacios litorales de Ciudad de La Habana y sus problemas ambientales}

La ciudad de La Habana, en lo fundamental se desarrolló a partir de una amplia cuesta de calizas miocénicas, que se eleva desde el nivel del mar, hasta unos 40-60 metros de altura, conformada al menos por 3-4 nivel de terrazas marinas. La cuesta es estrecha y relativamente inclinada al este, estando al extremo oeste conectada con un puente de cuesta que se une a una Ilanura interior calcárea. En este sector solo alcanza hasta los 30-40 metros de altitud y es más baja y poco inclinada. (Figura 1, Tabla I).

Diversos ríos pequeños alógenos cortan la cuesta. En el medio de esa llanura se forma la bahía de bolsa cerrada de La Habana . En la misma desembocan varios ríos. Por su carácter geoecológico, dicha cuesta se divide en cuatro sectores. Cada uno de dichos sectores, corresponde en general con un patrón de organización espacial, y una modalidad de ocupación, lo cual establece un conjunto de problemáticas espaciales y ambientales. De tal manera, cada uno de dichos sectores constituye un patrón al cual debe corresponder una determinada orientación de gestión.

La bahía de La Habana, constituyó el centro de atracción fundamental a partir del cual se desarrolló la ciudad de La Habana. El patrón espacial y arquitectónico implantado, es del estilo 


\section{ESPACIOS LITORALES DE CIUDAD DE LA HABANA}

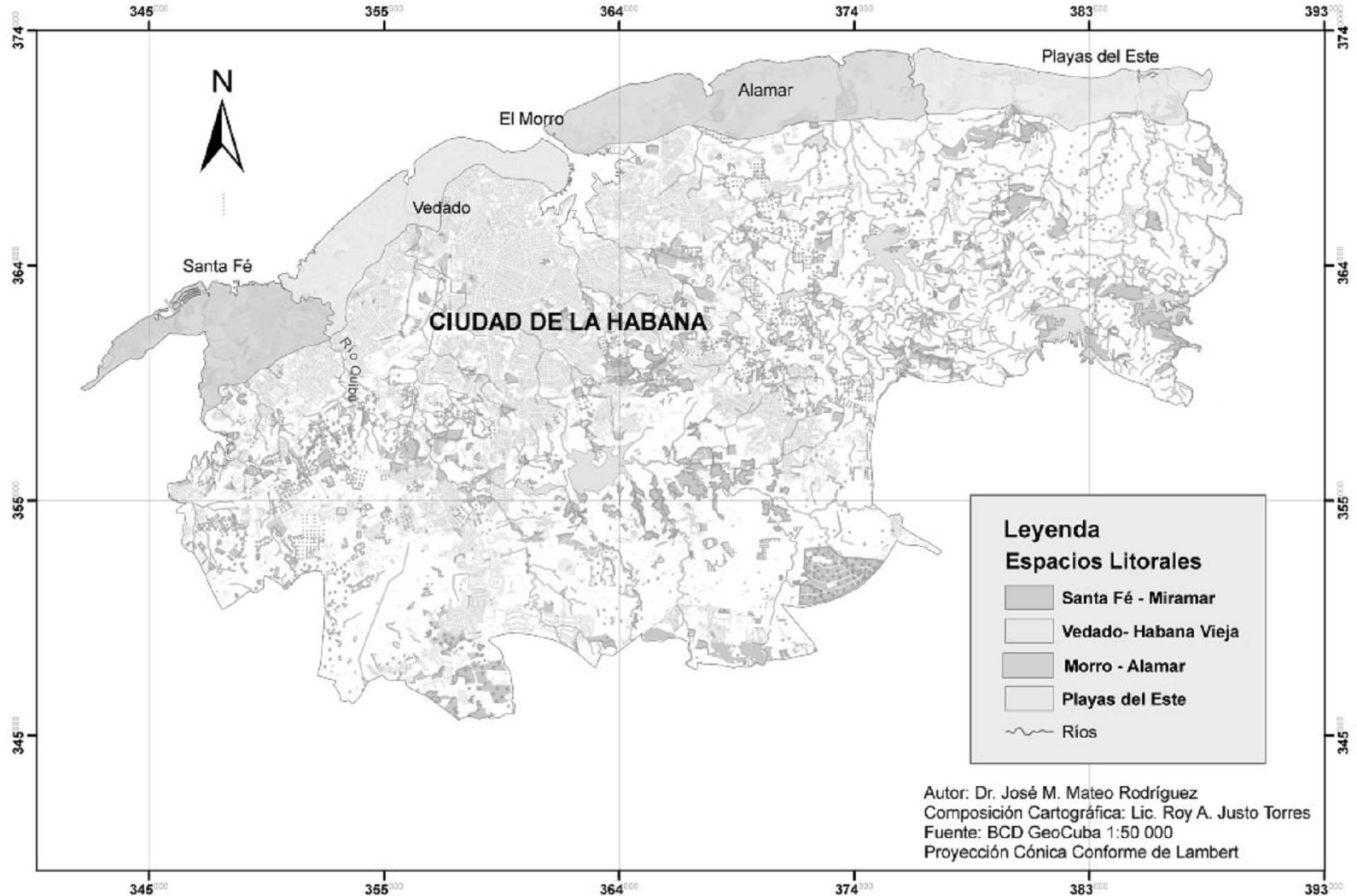

Figura 1. Mapa de espacios litorales de la Ciudad de La Habana

español clásico. La construcción de avenidas formadas por casas de columnas que forman verdaderos corredores públicos, correspondiente a los ejes principales, de casas tejas simples, sustituidas en lo fundamental por viviendas sólidas de cemento de puntual alto, conformaron la adaptación al ambiente tropical caribeño. La carencia total de arborización en calles y avenidas, era compensada por la presencia de parques que ocupan manzanas completas.

La expansión económica de comienzos de siglo $X X$, se manifestó no solo en la ampliación del modelo español, sino también en la conformación de un modelo de reparto de patrón medio con algunas variantes. La sustitución del corredor público, por casas y mansiones con portales de columnas, la conformación de áreas verdes en el entorno de las aceras, y de amplios parques, fueron rasgos distintivos del nuevo modelo. La ulterior expansión al oeste de la ciudad se conformo por repartos modernos y mansiones con amplias extensiones de parques y jardines particulares. El patrón en ajedrez, se sustituyo por un patrón de rasgos curvilíneos. (Coyula, 2001)

El acceso directo a la línea de la costa, solo se desarrollo en la parte centro oeste. En este sector también se formaron varios clubes, que no solo privatizaron la línea costea, sino 
TABLA I - Espacios litorales de Ciudad de la Habana.

\begin{tabular}{|c|c|c|c|}
\hline ESPACIO & $\begin{array}{l}\text { CARACTERISTI- } \\
\text { CAS FISICAS }\end{array}$ & PATRÓN ESPACIAL & $\begin{array}{c}\text { PROCESOS DE } \\
\text { OCUPACION ACTUAL }\end{array}$ \\
\hline $\begin{array}{l}\text { 1.-San ta Fe - } \\
\text { Miramar }\end{array}$ & $\begin{array}{l}\text { Terrazas marinas bajas, en } \\
\text { cuesta alargada, con } \\
\text { ensenadas y amplias } \\
\text { lagunas litorales, y playas } \\
\text { de cochas }\end{array}$ & $\begin{array}{l}\text { Parte periférica de los } \\
\text { repartos de patrón medio } \\
\text { alto y poblados litorales, } \\
\text { con clubes privad os en la } \\
\text { lín ea costera. }\end{array}$ & $\begin{array}{l}\text { Ruptura de la segregación espacial } \\
\text { y procesos embrionarios de } \\
\text { privatización; conurbación de } \\
\text { poblados }\end{array}$ \\
\hline $\begin{array}{l}\text { 2.-Vedado - } \\
\text { Habana Vieja }\end{array}$ & $\begin{array}{l}\text { Terrazas marinas, en } \\
\text { cuesta corta, con una bahía } \\
\text { en bolsa profunda }\end{array}$ & $\begin{array}{l}\text { Ciudad antigua de patrón } \\
\text { colonial y republicano, con } \\
\text { paseo cercado de edificios } \\
\text { (malecón) en la línea } \\
\text { costera }\end{array}$ & $\begin{array}{l}\text { Deterioro del fon do de viviendas, } \\
\text { socialización de vivien das, e } \\
\text { implantación de programa de } \\
\text { recuperación del patrimonio } \\
\text { arquitectónico de la ciu dad } \\
\text { colonial. }\end{array}$ \\
\hline $\begin{array}{l}\text { 3.- Morro - } \\
\text { Alamar }\end{array}$ & $\begin{array}{l}\text { Terrazas marinas, en } \\
\text { cuesta corta, con una bahía } \\
\text { en bolsa profunda, } \\
\text { atravesado por ríos que } \\
\text { forman cañones, y playas } \\
\text { en los deltas de cierre en la } \\
\text { desembocadura }\end{array}$ & $\begin{array}{l}\text { Fortalezas colon iales, y } \\
\text { pueblo litoral antigua; } \\
\text { nuevas urbanizaciones del } \\
\text { periodo revolucion ario en } \\
\text { forma de conjunto } \\
\text { habitacion ales. }\end{array}$ & $\begin{array}{l}\text { Urbanizaciones en conjuntos } \\
\text { habitacionales qu e corresponden } \\
\text { con la ocupación del periodo } \\
\text { revolucionario, quedando el pueblo } \\
\text { colonial englobado entre ellos. }\end{array}$ \\
\hline $\begin{array}{l}\text { 4.-Playas del } \\
\text { Este }\end{array}$ & $\begin{array}{l}\text { Cordón litoral arenoso, } \\
\text { con dunas y playas } \\
\text { continuas que bordean } \\
\text { lagunas litorales } \\
\text { conectadas con sistemas } \\
\text { fluviales que forman } \\
\text { cañones al atravesar a } \\
\text { cuesta. }\end{array}$ & $\begin{array}{l}\text { Repartos modern os, de } \\
\text { baja den sidad, y pueblo } \\
\text { litoral, asociado a repartos. }\end{array}$ & $\begin{array}{l}\text { Ruptura de la segregación espacial } \\
\text { y procesos embrionarios aun que } \\
\text { amplios de privatización; } \\
\text { conurbación de poblado; } \\
\text { ampliación del poblado litoral. }\end{array}$ \\
\hline
\end{tabular}

que también dio lugar a la construcción de muros en la duna y en la plataforma, que alteraron significativamente los procesos costeros.

La parte este de la ciudad, con excepción de los pueblos litorales, quedó prácticamente congelada, hasta los años 50 del siglo XX. En este sector, se comenzaron a erigir repartos modernos completamente privatizados y cerrados al acceso al público. Gran parte de la duna y la playa sufrieron de construcciones, y de la creación de plantaciones de árboles exóticos, que constituyeron obstáculos al oleaje y que condujeron a intensificar los procesos de erosión de la playa.
Los pueblos litorales, en los extremos este y oeste de la ciudad, se formaron por bungallows de madera y tejas rojas de arcilla. La ulterior expansión y conurbación de dichos poblados, ha dado lugar a la modificación e incluso desaparición parcial de ese patrón morfológico. Al mismo tiempo, se ha producido una expansión de los poblados con repartos de casas modernas y conjuntos habitacionales.

Gran parte de los espacios vacíos, tanto amplios como aquellos pequeños entre las clases de patrón alto, han sido rellenados por conjuntos habitacionales de viviendas populares construidos en el periodo revolucionario. Al menos tres modelos se han erigido: los de la ciudad socialista 
original, los de la urbanización generalizada y los de la urbanización del tipo de ciudades capitalistas. Sin embargo, la urbanización revolucionaria, en todos los casos ha tenido un rasgo común: conservar una franja de 0,5 a 1 kilómetro de ancho, que ha conformado un verdadero espacio costero vacío (Mateo, 2004).

\section{Procesos espaciales ocurridos en La Habana en los últimos 50 años}

Con el propósito de construir un nuevo escenario para la gobernabilidad, sustentada en bases socialistas, y para resolver los problemas ambientales y espaciales heredadas del antiguo modelo neocolonial, se han conformado en los últimos 50 años los siguientes procesos espaciales en el litoral urbanizado de Ciudad de La Habana. (Scarpaci et.al., 2002; Mateo, 2003)

- Creación de una nueva estructura espacial, con nuevas funciones y relaciones espaciales: La nueva estructura espacial, conservó en gran parte las áreas residenciales, aunque se han creado nuevas áreas y funciones. Entre ellas están: nueva zona industrial y de almacenamiento; nuevas zonas de recreación; parque urbano; nuevas zonas; empresarial, de negocios y comercial; zona de patrimonio histórico cultural; área con plantaciones forestales que desempeña un papel de amortiguamiento. El desafío consiste en consolidar esa nueva estructura y garantizar relaciones espaciales armónicas y coherentes (Coyula, 2005).

- Conurbación: Originalmente y prácticamente hasta el Triunfo de la Revolución La Habana, en si era una ciudad región, formada por varios centros y pueblos que tenían su autonomía propia. Desde hace 50 años se ha producido un fuerte proceso de conurbación, principalmente a través de ejes viales. La reforma política administrativa, que dio lugar a que La Habana conformarse una provincia, ha estimulado este proceso. Sin embargo, aun esos poblados tienen en parte una relativa singularidad. El desafío, es conservar esa singularidad, estimulando la formación de centros secundarios, en el contexto de la gran ciudad.

- Recuperación de las identidades de los lugares. Ligado a ese proceso de conurbación, está la perdida de identidad de muchos de esos lugares. Esa perdida se refleja en un proceso de no lugarización, en el que el antiguo paisaje y espacio es desvirtuado por formas existentes en todas las ciudades. Se pierde la singularidad, pero también la armonía entre el todo y las partes. Muchos de los nuevos habitantes, principalmente inmigrantes, no tienen relación afectiva con esos lugares. (Mateo, 2004). De lo que se trata es de potenciar la singularidad, por varias vías: divulgativas, paisajísticas, conservación del patrimonio y también una fuerte labor de educación geográfica y ambiental.

- Alteración de la segregación espacial: La Habana neo colonial, era profundamente segregada, espacial y ambientalmente. Las áreas litorales, en particular las del extremo oriental y occidental eran las de mejor calidad ambiental y las que albergaban a las clases pudientes. Los pobres, en particular negros y mestizos vivían en algunas áreas de manglar, en los fondos de los valles de ríos inundables, y al borde de la bahía. Después de la Revolución, se ha alterado profundamente esa segregación: al abandonar las clases pudientes del país, esas áreas se han transformado en áreas residenciales para diplomáticos, en zonas de negocios y empresas y también en áreas residenciales. Muchos espacios vacíos se ha rellenado de conjuntos habitacionales donde viven todos los grupos sociales. El desafío consiste en continuar con ese proceso, ya que paradójicamente aun subsisten algunos pocos focos de pobreza.

- Ruptura de la privatización del acceso a la línea costera y espacios públicos. Toda la zona de asimilación costera, posterior a 1940 
fue privatizada, siendo ocupada por clubes, barrios cerrados privatizados, o casas con muros que limitaban el acceso al mar. Después del Triunfo de la Revolución, se cambió radicalmente esa situación: los clubes privados se convirtieron en círculos sociales obreros; las áreas cerradas se abrieron y se convirtieron en zonas residenciales. Aun quedan muchas viviendas con acceso limitado. El desafío actual consiste en ampliar cada vez más el acceso a toda la población de la zona costera.

- Completamiento de las estructuras espaciales y recuperación de fondos de viviendas y patrimonios. El proceso de verticalización, que había comenzado sobre todo en el barrio del Vedado en los años 50, fue prácticamente interrumpido. Más que todo se han creado nuevas urbanizaciones en espacios anteriormente vacíos. Sin embargo, gran parte del patrimonio arquitectónico, paisajístico e histórico quedo abandonado, perdiéndose o afectándose el fondo de edificaciones. En la Habana Vieja, se ha establecido un plan singular de recuperación del casco histórico, con presencia de la población que allí vive. El desafío es continuar con ese proceso, caro y complejo.

- Construcción de nuevas formas simbólicas, y de hitos en el paisaje. La Habana de antes de la Revolución estaba inundada de propagando comercial. Los monumentos se dedicaban a los héroes de la independencia, aunque también habían algunos dedicados a caudillos y políticos de la Republica Neocolonial. La propaganda y divulgación, es en los momentos actuales fundamentalmente política. La divulgación comercial casi no existe. La polución visual es mínima. Algunos monumentos de políticos, han sido eliminados o sustituidos. Junto con monumentos de patriotas por la independencia, han sido creados nuevos monumentos, dedicados a héroes de la Revolución. La Plaza Cívica, no culminada en tiempos de la republica neocolonial, se convirtió en la Plaza de la Revolución. Muchos edificios referenciales cambiaron de función: palacios en museos; cuarteles en escuelas. El desafío es continuar con ese proceso, y ganar en calidad en las representaciones simbólicas en el paisaje (Correa, 2007).

- Revitalización de recursos y animación espacial. La animación de La Habana pre revolucionaria, se vinculaba a áreas nocturnas, a la actividad comercial privada, a algunas áreas de recreación muchas de ellas cerradas a la mayoría. La Revolución socializo los recursos para el pueblo. Sin embargo, se cerraron muchas instalaciones, y la animación de la ciudad, privada de las luces de la propaganda del capital, en muchas ocasiones quedo apagada. El desafío es recuperar esa animación; es potenciar el uso de los recursos del litoral, tal vez abriendo la posibilidad a la iniciativa comunitaria e incluso privada, aunque con una visión social.

- Cohesión espacial en áreas de playa: en la parte oriental de la ciudad, existe la zona de las Ilamadas "Playas del Este". Consiste en una extensión de playas y dunas con excepcionales recursos para el baño de mar y de sol, circundados por una amplia extensión de terrazas marinas llanas. Antes del Triunfo de la Revolución, el extremo oriental de esa área era explotada por la población local para garantizar la recreación de la población común. Toda la parte occidental, era usufructo de barrios residenciales cerrados y privados. Sin embargo, el proceso de ocupación era poco avanzado, y la urbanización de muy poca densidad. La Revolución cambio drásticamente esa situación. Por increíble que parezca, esa área, que en condiciones de económica de mercado hubiese sido densamente ocupada y apropiada por la especulación inmobiliaria, fue desde hace 50 años, prácticamente congelada. Con excepción del Hotel "Mar Azul", prácticamente no se han realizado nuevas edificaciones. En general, esa área es usada 
en lo fundamental para el turismo internacional, pues los edificios y casas de la burguesía se convirtieron en casas hoteleras. Su uso para tal función, es dirigida de manera indirecta, a través de la transportación publica, la cual es dirigida en lo fundamental al parte mas oriental, ocupada por el antiguo poblado litoral de Guanabo. Tal vez, en un futuro se piense en hacer nuevas ocupaciones. El desafío será conservar a toda costa la calidad ambiental y paisajística de la zona expuesta, que sea dirigida por un diseño coherente y riguroso de Ordenamiento Ambiental.
- Recuperación de interfases naturales, y re naturalización de objetos (Tabla II). Incluye: La construcción de muros, edificios prácticamente en la línea costera, que ha dado lugar a un intenso proceso de erosión y abrasión marina, y a la perdida de la mayor parte de los sistemas litorales (lagunas, dunas, vegetación litoral). En los últimos años, se observa en algunos lugares un intenso proceso de penetración del mar, sobre todo en ocasión de eventos significativos tales como frentes $y$ huracanes. Ello está dando lugar a un proceso de acumulación de arena, en

TABLA II - Problemas ambientales de los espacios litorales de ciudad de la habana

\begin{tabular}{|c|c|c|}
\hline ESPACIO & $\begin{array}{r}\text { PROBLEMAS } \\
\end{array}$ & POSIBLES SOLUCIONES \\
\hline 1.-Santa Fe -Miramar & $\begin{array}{l}\text { - Falta de acceso a la costa } \\
\text { - Erosión y abrasión costera } \\
\text { - Acumulación en post duna y can ales } \\
\text { - Desagüe de canales de aguas negras } \\
\text { - Espacios vacíos en la línea costera }\end{array}$ & $\begin{array}{l}\text { - Recuperación de dunas y draga do de } \\
\text { canales } \\
\text { - Demolición de objetos, situados en la línea } \\
\text { costera } \\
\text { - Revitalización de objetos y movilización } \\
\text { de recursos. } \\
\text { Obras hidrotécnicas para limpieza de } \\
\text { desagües }\end{array}$ \\
\hline $\begin{array}{l}\text { 2.-Vedado - Habana } \\
\text { Vieja }\end{array}$ & $\begin{array}{l}\text { - Deterioro de las edificaciones en el } \\
\text { prime frente del mar. } \\
\text {-Inundaciones y penetraciones del mar } \\
\text { ocasionales. } \\
\text { - Contaminación de la primera terraza } \\
\text { abrasiva. }\end{array}$ & $\begin{array}{l}\text { - Recuperación del fondo arquitectónico. } \\
\text { Implementación de obs hidrotécnicas para la } \\
\text { evacuaron del drenaje. } \\
\text { - Limpieza del frente costero }\end{array}$ \\
\hline 3.- Morro - Alamar & $\begin{array}{l}\text {-A cumulación y contaminación post } \\
\text { playa de las dunas de los deltas de } \\
\text { cierra. } \\
\text {-Abandono y deterioro de obras en la } \\
\text { primea terraza. } \\
\text { Vacíos de uso en la primera terraza, con } \\
\text { crecimiento de matorrales. }\end{array}$ & $\begin{array}{l}\text { - Recuperación de objetos y obras } \\
\text { - Limpieza y rehabilitación de la vegetación } \\
\text { de la primera terraza y de las playas de los } \\
\text { deltas. }\end{array}$ \\
\hline 4.-Play as del Este & $\begin{array}{l}\text { - Sub utilización del poten cial } \\
\text { - Erosión de playas y dunas } \\
\text { - Inundaciones en la primera terraza por } \\
\text { falta de desagüe. } \\
\text {-Deterioro de viviendas y objetos. } \\
\text {-Contaminación y suciedad de la playa. } \\
\text {-Acumulación de arena en canales y } \\
\text { calles. . }\end{array}$ & $\begin{array}{l}\text { - Revitalización y construcción de objetos } \\
\text { para la explotación turística. } \\
\text { - Demolición de objetos y casas construidos } \\
\text { en la playa y la duna. } \\
\text {-Construcción de obras hidrotécnicas para } \\
\text { estimular el desagüe de calles. } \\
\text { Fijación de dunas. } \\
\text { Limpieza de las playas. }\end{array}$ \\
\hline
\end{tabular}


playas, dunas, canales y desembocaduras de ríos. También se ha reforzado la resaca y la corrosión marina en los edificios situados en frente al mar. En algunas ocasiones dicha acumulación de arena se acompaña de desechos de todo tipo. Sin embargo, sobre todo en las nuevas urbanizaciones construidas por la Revolución, el primer frente de mar, ocupado por la primera terraza, en ocasiones no ha sido ocupado, quedando verdaderos espacios vacíos. En los últimos años, dichos espacios están siendo ocupados de manera casi espontánea, por áreas de agricultura, y pastos muy degradantes, por basureros o matorrales. El desafío consiste en una recuperación integral de esa primera terraza. En algunos casos serán necesario demoliciones, en otros casos verdaderos planes coherentes de ocupación y rehabilitación.

\section{Consideraciones finales}

La gobernabilidad espacial y territorial, correspondiente con la necesidad de orientar los cambios para construir el nuevo proyecto socialista, ha entrado parcialmente en contradicción con la capacidad operativa para orientar y estimular los cambios, o sea con la governanza. Los siguientes factores han sido los responsables de esa contradicción (Coyula, 1998):

- La carencia de recursos
- La espontaneidad, el voluntarismo y a superficialidad en la toma de algunas decisiones.

- La carencia de estudios integrales y trabajos coherentes de planificación ambiental y espacial.

- La incipiente, fragmentada y a veces incoherente gestión.

- Un pobre manejo de los cambios ambientales y espaciales que ocurren en los espacios litorales.

Ha sido ingente y trascendental la tarea de paralizar y obstaculizar el avance de un modelo capitalista perverso que se había comenzado a instalar en La Habana a comienzos de los años 50. Sin embargo, el nuevo modelo espacial de ocupación y apropiación, si bien coherente con una nueva concepción de la organización social ha respondido mas que todo a medidas fragmentadas, espontáneas y desarticuladas. Después de 50 años, se precisa retomar el nuevo modelo original de ocupación socialista ya basado en el conocimiento y la experiencia de gestión popular y participativa de casi 50 años.

Los nuevos modelos de desarrollo regional y local que se construyen en América Latina pueden aprender mucho de la experiencia cubana. Se trata por tanto, de analizar y divulgar esas experiencias, para que con sus luces y sus sombras, puedan ser una de las fuentes en que beban los nuevos proyectos sociales, espaciales y ambientales que se construyen en la región.

\section{Bibliografía}

CORRÊA, R. L. O espaço urbano. São Paulo: Editora Ática, 4a edição, 2000.

Uma sistematização da análise de monumentos na Geografia. Revista Terr@ Plural, 2007, 1 (1): 9-22p.

COYULA, M. Ambiente urbano y participación en un socialismo sustentable. Temas (1997): no 9; 54-61p.
COYULA, M. El futuro de la ciudad: Desarrollo en equilibrio. Tareas, Revista del Centro de Estudios Latinoamericanos, Panamá, No. 107, (2001), 27$40 p$.

. Repensar La Habana. Revista Comunidad, Instituto de Planificación Física, Habana, Cuba.; (2005): no 4, 98-103p.

MATEO, J. Paisajes Naturales y Culturales de Cuba: cambios ocurridos en los últimos 50 años. 
Encuentro de Geógrafos de América Latina, 9., 2003, Mérida, Espanha. Atas... Mérida (2003). 1 CD.

MATEO, J. Sostenibilidad espacial y ambiental de la Provincia de Ciudad de La Habana. Informe de los resultados del Proyecto CAESAR. La Habana, mimeo. 2004, 68p.

OCHOA, E. e OLSEN, S.B. Elementos para preparar una línea de base de gobernabilidad. Encontro Regional sobre Humedales Costeros Patagónicos, 2007, Buenos Aires, Argentina.
Atas... Proyecto GEF Marino ARG 02/018 GEF/ PNUD; Buenos Aires, 9-11p. Disponível em http:/ /www.ecocostas.org/images/documentos/ 11 tallerhumedalescosterospatagonicos.pdf

SANTOS, M. A natureza do espaco. Técnica e tempo. Razão e emoção. São Paulo: Editora Hucitec, 1996.

SCARPACI, J.L.; SEGRE, R. e COYULA, M. Havana: Two faces of the Antillean Metropolis. California: The University of North Carolina Press, 2002.

Trabalho enviado em outubro de 2009 Trabalho aceito em novembro de 2009 